\title{
Martensite Variant Identification For Shape Memory Alloys By Using Graph Neural Networks
}

\section{YI-Ming Tseng}

Department of Materials Science and Engineering, National Chiao Tung University

\section{Pei-Te Wang}

Department of Materials Science and Engineering, National Chiao Tung University

Nan-Yow Chen

National Center for High-Performance Computing

An-Cheng Yang

National Center for High-Performance Computing

Nien-Ti Tsou ( $\nabla$ tsounienti@nctu.edu.tw)

Department of Materials Science and Engineering, National Chiao Tung University

\section{Research Article}

Keywords: SMAs, molecular dynamics (MD), nanoindentation process, orthorhombic, martensitic transformation

Posted Date: January 26th, 2021

DOl: https://doi.org/10.21203/rs.3.rs-152556/v1

License: (c) (i) This work is licensed under a Creative Commons Attribution 4.0 International License.

Read Full License 


\title{
Martensite Variant Identification for Shape Memory Alloys By Using Graph Neural Networks
}

\author{
Yi-Ming Tseng ${ }^{1}$, Pei-Te Wang ${ }^{1}$, Nan-Yow Chen ${ }^{2}$, An-Cheng Yang ${ }^{2}$, and Nien-Ti Tsou ${ }^{1, *}$ \\ ${ }^{1}$ Materials Science and Engineering, Hsin-Chu, 300, Taiwan \\ ${ }^{2}$ National Center for High-performance Computing, National Applied Research Laboratories, Hsin-Chu, 300, Taiwan \\ *tsounienti@nctu.edu.tw
}

\section{ABSTRACT}

Detailed microstructure evolution in shape memory alloys (SMAs) is typically studied by molecular dynamics (MD) simulations. However, the conventional post-processing tools for atomistic calculations, such as CNA and PTM, fail to identify distinct crystal variants and to reveal twin alignments in SMAs. In the current work, a powerful and efficient post-processing tool based on GraphSAGE neural network is developed, which can identify multiple phases in martensitic transformation, including the orthorhombic, monoclinic and $\mathrm{R}$ phases. Where the network was trained by the results of sets of temperatureand stress-induced martensitic transformation MD calculations. The accuracy and generality were also verified by the application to the cases which did not appear in the training dataset, such as unrecoverable nanoindentation process. The proposed method is rapid, accurate, and is ready to be integrated with any visualization tool for MD simulations. The outcome of the current work is expected to accelerate the pace of atomistic studies on SMAs and related materials.

\section{Introduction}

Shape memory alloys (SMAs) are intelligent materials which have been widely used in aerospace engines $^{1,2}$, biomedical materials ${ }^{3}$, and actuators ${ }^{4}$ in the recent years. They have unique mechanical behavior and properties due to their fascinating microstructures and phase transformations. When SMAs are at high temperature subjected to an external load or a cooling process, unit cells undergo phase transformation from the austenite phase with high symmetry to the martensite phases with lower symmetry. A crystal system with lower symmetry in SMAs typically has several ground states with equal energy, known as crystal variants. Reorientation of variants dominates the SMAs mechanical properties, such as superelasticity ${ }^{5,6}$, shape memory effect ${ }^{7,8}$, and remarkable damping capacity ${ }^{9}$. The reorientation of variants is mainly achieved by the movement of these interfaces between variants with excellent mobility ${ }^{10}$. Thus, in order to understand the detail of the reorientation of variants, it is crucial to identify distinct phases/variants and track the movement of their boundaries.

These tasks are typically difficult in experiments and usually studied by molecular dynamics (MD) simulations. Recently, several conventional microstructure identification methods, such as $\mathrm{CNA}^{11-13}$ and $\mathrm{PTM}^{14}$, have been proposed which visualize the results of MD to allow us understand the internal interactions and microstructure evolution of materials. They help researchers identify crystal systems such as BCC, FCC, and HCP in an elegant and efficient way. For example, Srinivasan ${ }^{15}$ applied CNA to identify the microstructure evolution in NiTi alloy due to the stress-induced phase transformation simulated by MD. Where the interfaces between BCC and HCP can be clearly observed. However, it is still difficult to identify distinct crystal variants and to reveal their alignments in NiTi alloys. This is because the BCC structure may include Austenite and Rhombohedral crystal system, while HCP structure may include 
Orthorhombic and Monoclinic crystal system ${ }^{16}$.

Therefore, Yang and Tsou ${ }^{17}$ proposed Martensite Variant Identification Method (MVIM) to identify multiple variants occurred in SMAs, by examining the lattice transformation matrices. Lu et. al ${ }^{18}$ and Chen and Tsou ${ }^{19}$ used MVIM to successfully link the microstructure evolution with mechanical properties such as superelastic effects. In the current work, MVIM was further improved by homogenizing/smoothing the singularity due to the thermal vibration. This gives more clear images of the resulting microstructures. However, the traditional matrix operation methods are the bottle neck of the efficiency of calculation, when dealing with a system with a large amount of atoms. Therefore, the current work aims to develop a highly-efficient crystal variants visualization method based on Graph neural network.

Graph neural network ${ }^{20}$ has excellent reasoning ability for graphical structured data which consists of vertices and edges. The features of each vertex are aggregated with those extracted from its adjacent vertices via edges during the forward propagation process. This local connection in graph neural network prevents each vertex being affected by the information of vertices without immediate connections, reducing the complexity of computation compared with the fully connected neural networks. It is of interest that molecular spaces can also be analog to a graph ${ }^{21}$, where each atom can be regarded as a vertex of the structure in the graph while each bond between a pair of adjacent atoms can be regarded as edges. Several successful examples of the application of Graph neural network to molecular spaces include the prediction of drug response ${ }^{22}$ and chemical reactivity ${ }^{23}$, showing high accuracy and computational efficiency.

Thus, GraphSAGE ${ }^{24}$, which is one of the most efficient and general graph neural networks, was applied here to replace MVIM as a powerful MD post-processing tool, visualizing crystal variants in SMAs. GraphSAGE can transfer topological information of the entire molecular space, including distortion of unit cells, into a graph structure, and thus, cumbersome calculations of matrix can be saved. The crystal variants can then be quickly identified. This method, named MVIM-G, is ready to be integrated with OVITO $^{25}$, which is the most popular visualization and analysis tool for MD simulation. The detailed alignment between austenite and martensite phases and the classification of distinct variants in SMAs can be revealed in detail.

\section{Results}

\subsection{Prediction of MVIM-G in training dataset}

Figure 1 shows the selected post-processed MD microstructures under different boundary conditions (listed in Table 4) by three different methods, i.e. the conventional CNA, MVIM, and the proposed GraphSAGE version of MVIM (MVIM-G). Where the cutoff in CNA was set as the lattice length of the Ni-Ti alloy. Figure 1(a)-(c) show a MD snapshot of a NiTi SMA undergone a stress-induced martensitic transformation at $500 \mathrm{~K}$ (corresponding to Case 1 described in Table 4). It can be observed that CNA classified the entire crystal as HCP and two unknown interfaces (identified as Other), while MVIM can distinguish $O 1$ and $O 2$ from the regions of $\mathrm{HCP}$, giving a striped twin structure. It is of interest that there are some austenite $A$ classified along those interfaces. It is because that the symmetry of the distortion of $O 1$ and $O 2$ across the interfaces confines the corresponding transform matrices, resulting in the reference matrix. Figure 1(c) shows the result of the current MVIM-G, having very good agreement with it of MVIM. It is worth noting that the interfaces identified by MVIM-G is sharper than those identified by the other two methods. It is because the graph neural network aggregates neighbor information providing a more homogeneous result, especially at the junction/interface between distinct variants. Although this procedure may compromise on the level of one-by-one accuracy of the model, the efficiency of the classification 
can be significantly improved and, after all, the focus of the visualization is the overall microstructure in SMAs rather than specific atoms in the region of ambiguity. This phenomenon can also be found in the following cases.

Figure 1(d)-(f) illustrate Case 2, where the crystal subjected to a compressive load along the [010] direction. It can be found that MVIM and MVIM-G successfully identified the self-accommodated rank-two herringbone twin structure composed by four monoclinic crystal variants $(M 5-M 8)^{26}$ that were energetically favored by the load. It is remarkable that $O 3$ and $O 4$ are also identified in the regions near interfaces as shown in Figure 1(e)(f). The two variants served as transitional phases during martensitic transformation, and this phenomenon is also reported in the literature ${ }^{27}$. Although CNA fails to identify such detailed variant arrangement, it can still identify FCC (marked green lines in Figure 1(d)), where the lattices are not martensite phases. Thus, these lattices were assigned with the most similar variants with the closest distortions by MVIM and MVIM-G.

Cases 3-5 are temperature-induced simulations in different axial-coupling settings, as shown in Table 4. Figure 1(g)-(o) show the formation of a rank-3 herringbone twin structure mainly composed by 8 distinct crystal variants. This indicates that results of temperature-induced simulations are particularly suitable to serve as training dataset, since they can produce a more complex structure with the present of multiple variants compared to the cases of stress-induced martensitic transformation. In addition, the microstructures in the three cases are, in fact, equivalent by rigid body rotation, allowing the model learn the relationship between the nature of symmetry between variants. The three cases not only trained the proposed GraphSAGE with the advantage of data augmentation, but also gave a more realistic behavior of atoms compared with artificial rotation of the data since a randomness thermal fluctuation of atoms were included here. In Figure 1(g)-(o), the results of MVIM-G show more austenite $A$ and less Other along the interfaces compared with those of MVIM. It is of interest noting that the former had an even better agreement with those of CNA, where most of the regions along the interfaces were identified as BCC (corresponding to phase A), compared to the later. This is because, in MVIM, Other is a phase which does not belong to any of 23 crystal variants, giving inconsistent features or criteria for the corresponding classification, and this makes the MVIM-G temps to identify a given lattice by the 23 types of phase rather than Other and the results are typically influenced by the dominant variants in that local region. Although this gives a more clear and homogeneous post-processing results, some details in a very fine microstructure may be compromised. An example can be seen in the red circle in Figure 1(1) showing several missing segments of fine twins. 


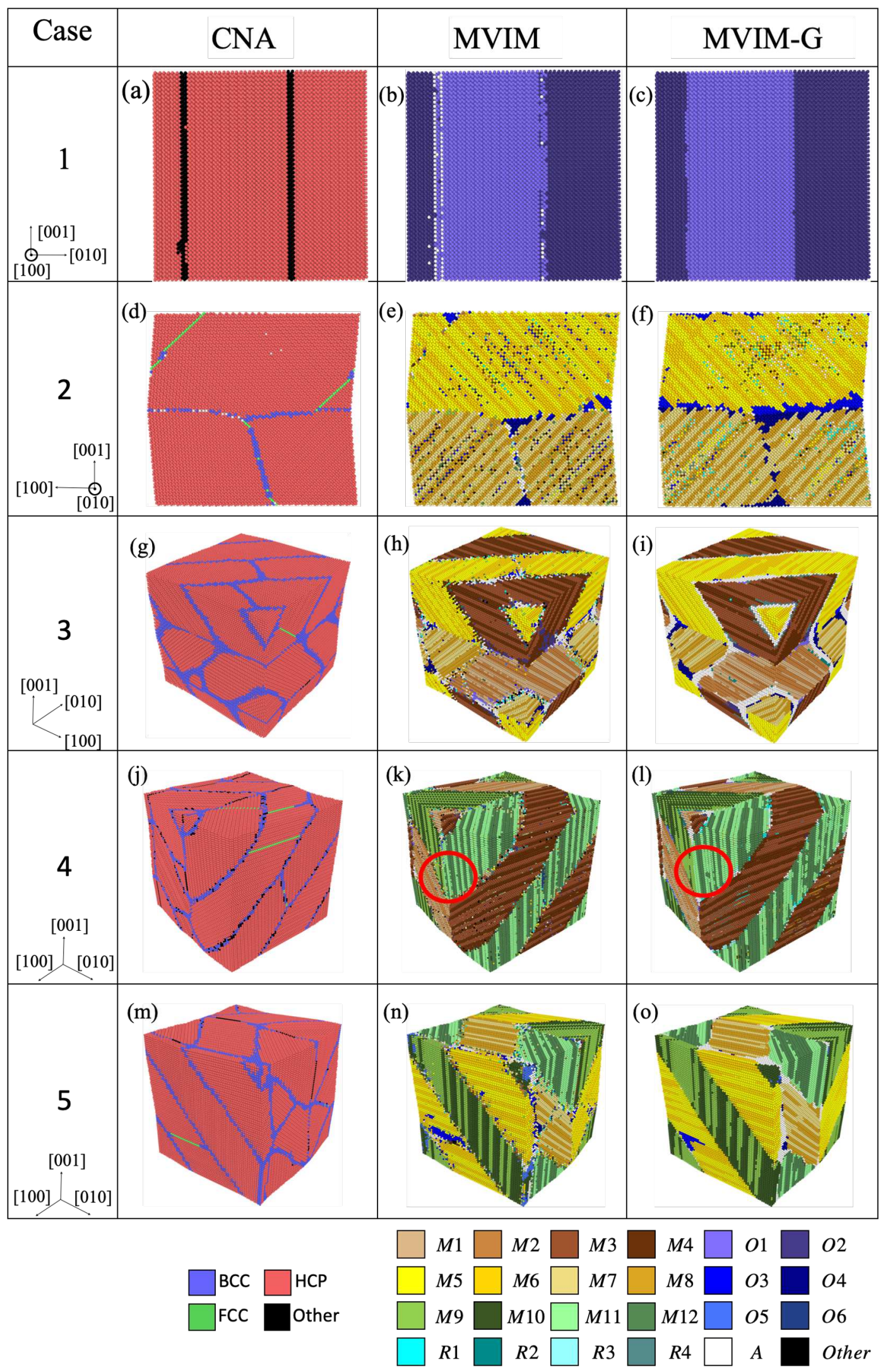

Figure 1. Selected snapshots for cases 1-5 post-processed by CNA, MVIM and MVIM-G. 


\subsection{Prediction of MVIM-G on unseen cases}

\subsubsection{Dataset and simulation setting}

Now consider the cases of temperature-induced and stress-induced martensitic transformation in SMAs that have not appeared in the training data, in order to demonstrate the versatility of the current MVIM-G model. Here, 2NN-MEAM potential energy ${ }^{28}$ was adopted by the simulation for both cases. The temperature-induced model was controlled by the isothermal-isobaric canonical ensemble (NPT) with pressure of 1.013 bar. The periodic boundary condition (PBC) was applied in all directions. The model was firstly relaxed at $0 \mathrm{~K}$ by the conjugate gradient method to achieve energy minimization and dynamic equilibrium. Then the initial velocity of the atoms at $500 \mathrm{~K}$ was assigned by Gaussian distribution for thermal equilibrium. This is because $500 \mathrm{~K}$ is well above the austenite finish temperature for NiTi SMAs, enabling a stable initial structure of austenite phase. After thermal equilibrium, the model was cooled from $500 \mathrm{~K}$ to $100 \mathrm{~K}$, and then gradually heated from $100 \mathrm{~K}$ to $325 \mathrm{~K}$, resulting in a stable microstructure with martensite phases.

For the stress-induced case, a nanoindentation process which resulted in an unrecoverable plastic deformation at the surface of a NiTi crystal was simulated. Here NPT ensemble was used, and the energy minimization process was the same as it in the temperature-induced case. The periodic boundary condition was applied in [100] and [010] directions only, and the free surface was applied in the positive [001] direction. The temperature $325 \mathrm{~K}$ of thermal equilibrium is consistent with the temperature selected in the literature ${ }^{15}$, which could obtain a stable martensitic transformation between austenite and martensite phases. The indenter used here was with radius of $30 \AA$ located $3 \AA$ above the (001) plane to avoid the overlap of the indenter and the crystal. Next, the indenter moved downward with a constant rate $\left(1.5 \times 10^{-4}\right.$ $\AA$ / femtosecond) until the indentation depth reaches $30 \AA$. Finally, the indenter was unloaded with the same rate. The results generated by the both cases were then post-processed by the CNA, MVIM, and the proposed MVIM-G model.

\subsubsection{Model validation}

Figure 2 details a selected microstructure at $325 \mathrm{~K}$ after the isobaric temperature-induced martensitic transformation process mentioned above. Here the image was replicated along the [100] and [010] directions for illustration purpose. It can be observed that the identification results of the three methods are highly consistent, as shown in Figure 2(a)-(c), showing high applicability of the current MVIM-G model to unknown dataset. This microstructure has good agreement with it reported in ${ }^{29}$ with the same model setting. Several interesting features in the SMA crystal can be discovered. Firstly, SMA undergone this specific process can produce a dart-shaped variant arrangement consisting of mainly six monoclinic phases, $(M 3, M 4),(M 7, M 8)$, and $(M 9,10)$, where the brackets indicate that the pair of variants having the same distortion axes. Secondly, it is of interest that the junction of these pairs of monoclinic phases maintained the austenite phase due to the mutual confinement of these three pairs of monoclinic phase which distorted along [100], [010], and [001], respectively. Thirdly, all the three post-processing methods gave rough interfaces between two pairs of monoclinic phases. Where CNA identified them as BCC, which could be the austenite phase or rhombohedral phase, intersected with Other; MVIM identified them as rhombohedral and monoclinic phases; the MVIM-G presents more rhombohedral phases on those interfaces. This result demonstrates the difficulty of all the current post-processing methods on identifying sharp interfaces in complex microstructures. Finally, by comparing Figure 2(b)(c), an obvious classification error made by the MVIM-G can be observed. That is, the small segments of blue lines (O5), marked by red circles, which is expected as the transition phase from $A$ to $M 9$ or $M 10$. However, the MVIM-G did not identify those segments from the matrix of $M 10$ successfully. This is because the amount of training data related to the phase transition interval between the orthorhombic and monoclinic 
phases was relatively less compared to it of other phases.

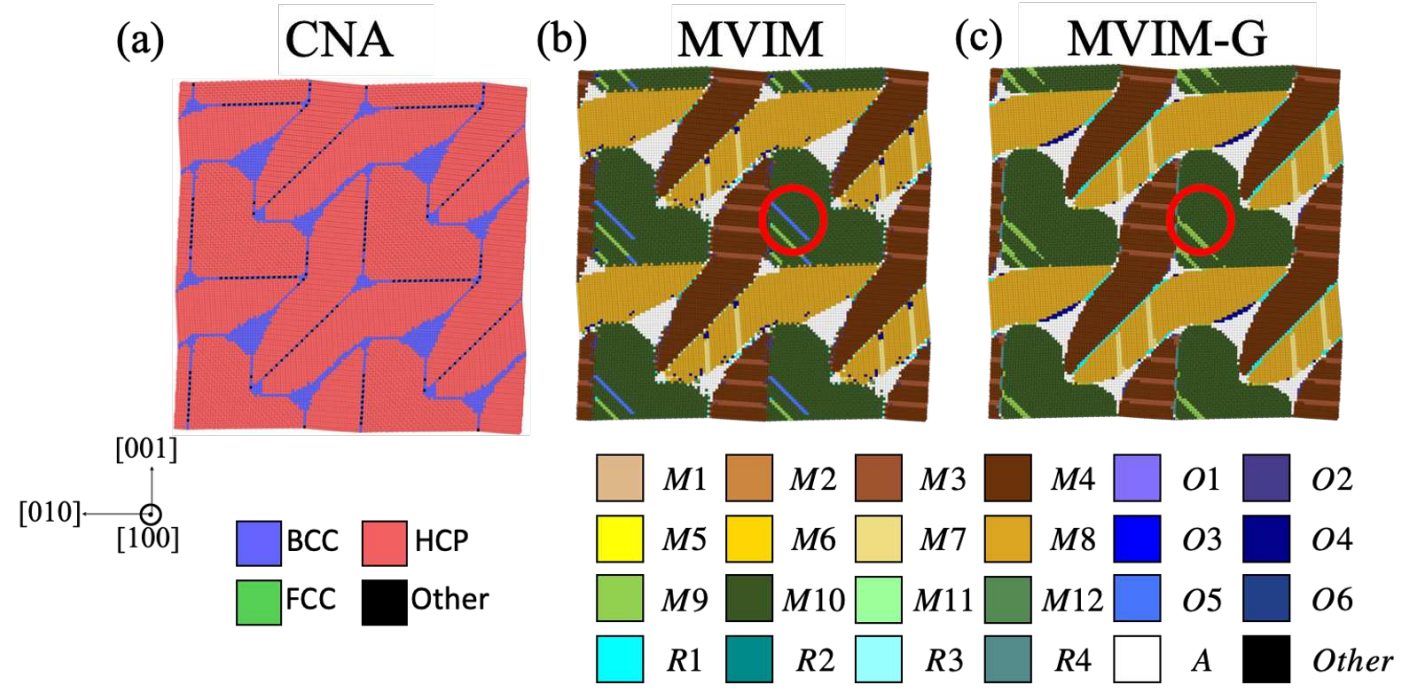

Figure 2. Selected snapshots for the case of isobaric temperature-induced martensitic transformation at $325 \mathrm{~K}$ post-processed by CNA, MVIM and MVIM-G.

Figure 3 shows the microstructural evolution in the (001) cross-section plane at half height of the crystal under the nanoindentation process. It can be observed that, again, the results generated by CNA, MVIM, and MVIM-G had good agreement, even when the case was not included in the training dataset, and with large plastic deformation and distortion of unit cells. Initially (indent depth $\delta=0 \AA$ ), the crystal adopted austenitic phase (parent phase). Figure 3(b)(c) show the microstructure consisting of the monoclinic and orthorhombic crystal variants when $\delta=15 \AA$. Where $M 9$ and $M 10$ around the center of the cross-section were induced by the concentrated stress due to the tip of indenter. The remaining part of the crystals adopted the transitional $O 5$ and 06 , as the stress was not sufficient to complete the full transformation from the austenite to monoclinic phases. It can be observed that the result of MVIM-G is smoother than that of MVIM. However, similar to the limitation mentioned above, some regions identified as $O 5$ and $O 6$ by MVIM were identified as $M 9$ and $M 12$ by MVIM-G. This problem can be solved by incorporating more data at such conditions in the training data set. Finally, the indenter reaches the maximum depth $\delta=30 \AA$, the phase transformation completed, giving a self-accommodated rank-2 herringbone twin structure consisting of M9-M12 monoclinic variants, as shown in Figure 3(e)(f). 


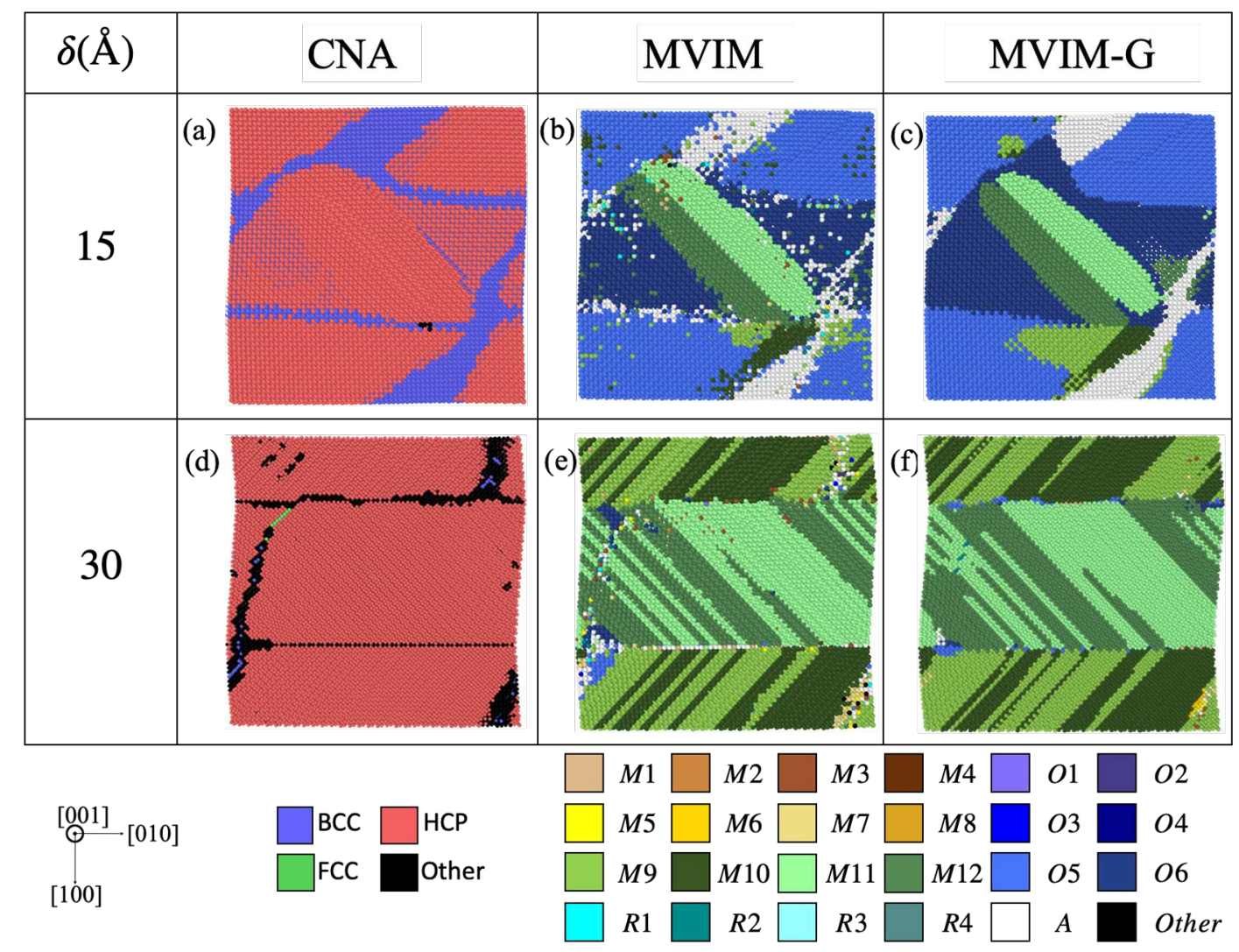

Figure 3. Selected snapshots at the cross-section plane of (001) for the case of nanoindentation when the indent depth $\delta=15,30 \AA$ A post-processed by CNA, MVIM and MVIM-G.

Now the accuracy and applicability to the unknown data sets of MVIM-G were verified. MVIM-G now is proved to enable a rapid and more detailed analysis on microstructural evolution in SMAs. This can be demonstrated by Figure 4, where microstructure at each stage during the nanoindentation process was linked with the data point on the load to the displacement curve (P-L Curve). The cross-section was along the (100) plane by slicing the crystal in half. As shown in Figure 4(a), the initial stage, the crystal adopted the austenite phase in white. As $\delta$ increased, austenite phase firstly transformed into $O 6$ and then completed phase transformation as $M 11$ and $M 12$, giving a linear P-L curve. This gives a heart-shaped monoclinic phase region with a front of orthorhombic phase at the bottom, as shown in Figure 4(b). When $\delta=15 \AA$, the orthorhombic variants $(O 5, O 6)$ transformed from the austenite phase dominated the crystal as shown in Figure 4(c). From stage (c) to (d), most of the orthorhombic phases transformed into monoclinic phases, giving a yielding platform on the P-L curve. It is worth noting that, base on the results, $O 5$ and $O 6$ served as the transitional phases to $M 9, M 10$ and $M 11, M 12$, respectively. This feature had good agreement with it reported in literature ${ }^{30}$, where the base-centered orthorhombic also served as transitional phase to the monoclinic phases. It is also remarkable that this feature cannot be revealed by other commercial post-processing tools, and the proposed MVIM-G is able to identify it with high efficiency (detailed in the next section). As the phase transformation was completed, the P-L curve then grew linearly, and the microstructure almost remained unchanged from Figure 4(d) to (e)). 


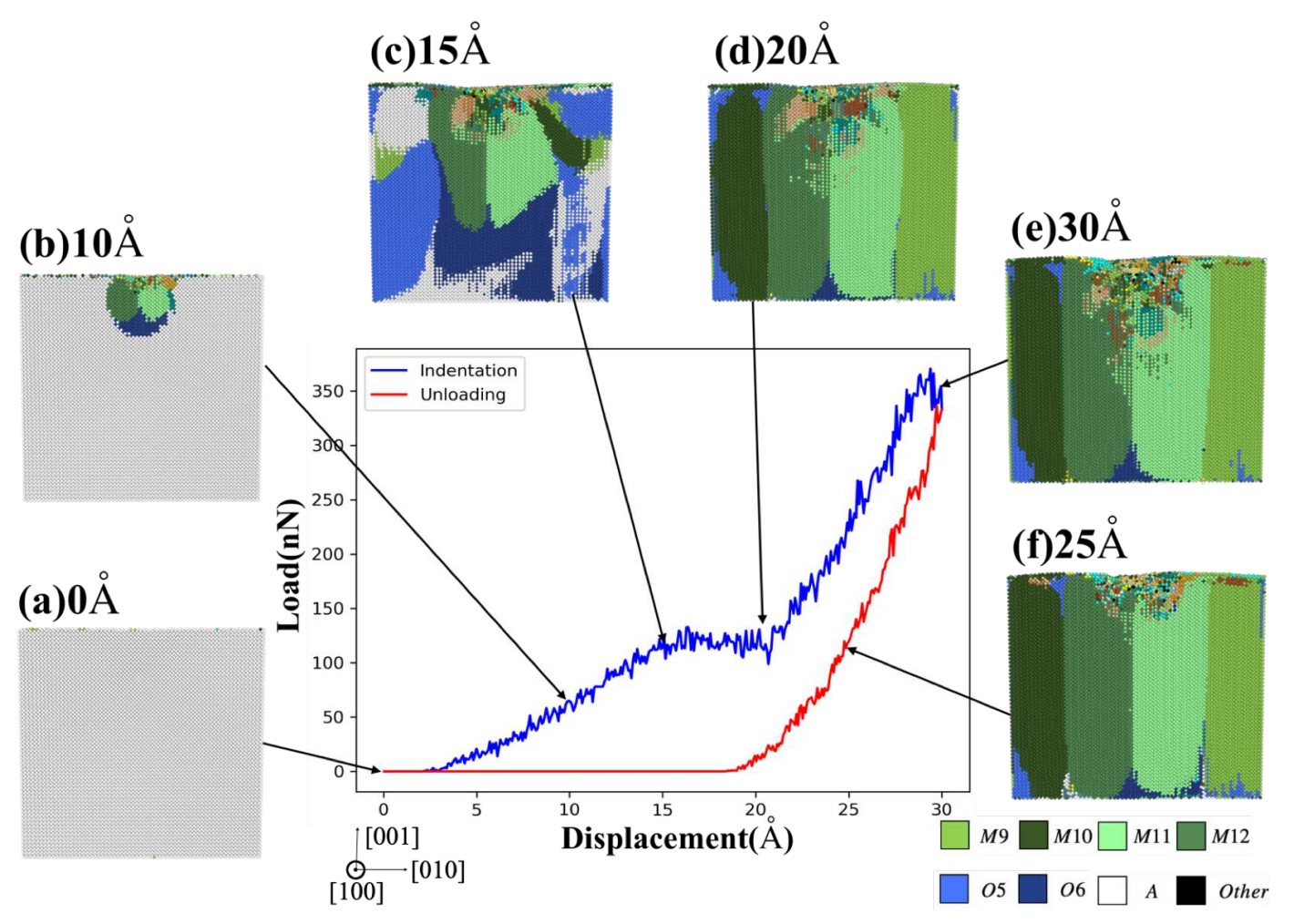

Figure 4. The P-L curve of NiTi SMAs during the nanoindentation process. The corresponding microstructures on the cross-section plane of (100) are post-processed by the MVIM-G.

The indenter was unloaded as $\delta=30$ Åwas achieved. Most regions in the microstructure, including the completely-transformed monoclinic region and the region close to the indenter tip, remained the same. However, the region further below the indenter tip with messy variant arrangement (consisting of $M 1, M 2$, $R 4$ etc.) returned to $M 9-M 12$ monoclinic variants, as shown in Figure 4(e) to (f). Finally, the indentation process left a residual deformation at the top of the crystal, and the microstructure did not return to the austenite phase due to the selected temperature $325 \mathrm{~K}$ between the martensite finish and start temperatures.

\subsubsection{Overall performance of MVIM-G}

Now consider the efficiency of the calculation of the current MVIM-G and the authors' previously proposed MVIM. Table 1 shows the average computing time required by the two methods when post-processing a given MD simulation result files outputted from the nanoindentation simulation mentioned above. Where 500094 atoms are included in that file. Although, MVIM-G required few more seconds to convert atom information into a special format of graph in the data preparation step, MVIM-G can significantly reduce the computing time for the phase classification tasks. Thus, MVIM-G enables a remarkable efficiency overall. It is worth noting that even we adopted three independent graph neural network models here and ensembled their results to complete the classification tasks for each atom, the demand of computing time was still in satisfaction.

The average training and testing accuracy of the MVIM-G model were $82.82 \%$ and $82.14 \%$, respectively. The values were obtained by compared the predicted variant by MVIM-G and the variant identified by MVIM method for each unit cell in the atomic models prepared by the procedures described in section 4 . Although the accuracy seems not to be fascinating, the misprediction was typically due to the inevitable complexity raised by thermal fluctuations, twin interfaces, free surfaces and highly distorted area, as discussed in the previous sections. Also, the purpose of the proposed method is for the visualization 
of atomistic calculations, revealing microstructures, patterns, and the overall distribution of the crystal variants and phases. Thus, with such high efficiency, MVIM-G is expected to be a handy post-processing tool to study the microstructural evolution during martensitic phase transformation in SMAs and related materials.

\begin{tabular}{|c||c|c|c|}
\hline \hline Model Name & Data preprocess time (s) & Phase classification time (s) & Total time (s) \\
\hline MVIM & 97 & 348 & 445 \\
\hline MVIM-G & 109 & 3 & 112 \\
\hline
\end{tabular}

Table 1. Time consumption in data preprocess and phase classification for MVIM and MVIM-G.

\section{Discussion}

In the current study, we proposed a powerful and efficient post-processing tool based on GraphSAGE for the results of atomistic simulations. This tool is particularly suitable for identifying multiple phases in martensitic transformation, including the orthorhombic, monoclinic and R phases, in shape memory alloys. The underlying theory is based on the classical crystallography and the complicated matrix calculation is replaced by graph neural network, enabling significant acceleration on the phase identification process. The outcome of the current tool provides in-depth insight of microstructure evolution and detailed twin arrangement in SMAs. These details and information cannot be revealed by any other current commercial post-processing tools. With the advantages of the current tool mentioned above, though, some details in very fine microstructures may be compromised, due to the homogenization nature of graph neural network; some regions of transitional phases, such as the orthorhombic or R phases, may not be correctly identified due to the insufficient amounts of the training data of this type, and this problem can be resolved by incorporating more data for the training process. This tool is ready to be integrated with any visualization tool for MD simulation, such as OVITO, and boost the pace of researches related to SMA atomistic calculations.

\section{Methods}

\subsection{Martensite Variant Identification Method (MVIM)}

When the austenite phase in SMAs transformed into the martensite phases, the unit cells changed from the cubic system to the orthorhombic, monoclinic, or trigonal (R-phase) crystal systems consisting of certain crystal variants. In order to identify the variants from the three martensite phases, four types of reference unit cell for each target atom in the model should be examined. Fig. 5 shows that a target atom marked in red can be regarded as a part of the three tetragonal reference unit cells and a cubic reference unit cell. Where the three tetragonal unit cells shown in Fig. 5( a)-(c) can reveal the deformation of the monoclinic or orthorhombic variants along axes [100], [010], and [001] directions, respectively, while the cubic unit cell shown in Fig. 5(d) reflects that of the trigonal variants along the [111] direction. The distortion of each unit cell can be determined by the three position vectors colored in yellow. It can be described by a $3 \times 3$ deformation gradient matrix $\mathbf{F}(\mathbf{x})=\nabla \mathbf{y}(\mathbf{x})$ based on Cauchy-born rule ${ }^{31,32}$, where $\mathbf{x}$ and $\mathbf{y}$ are the three position vectors before and after deformation. The rigid body rotation of the lattice can be eliminated by polar decomposition ${ }^{33}$, giving the transformation matrix $\mathbf{U}=\sqrt{\mathbf{F}^{\mathrm{T}} \mathbf{F}}$. The transformation matrix for the four types of unit cell corresponding to the target atom can be obtained. 
These four transformation matrices then compared with the theoretical transformation matrix $\mathbf{U}$ of 23 possible crystal variants in all the four crystal systems $(A, O, M$, and $R$ ), which can be found in the literature ${ }^{32}$.

(a)

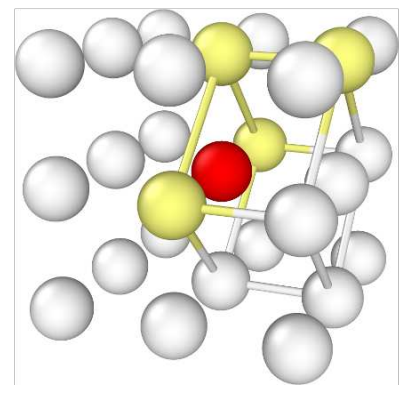

(b)

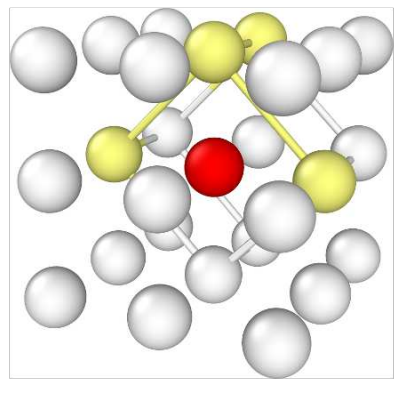

(c)

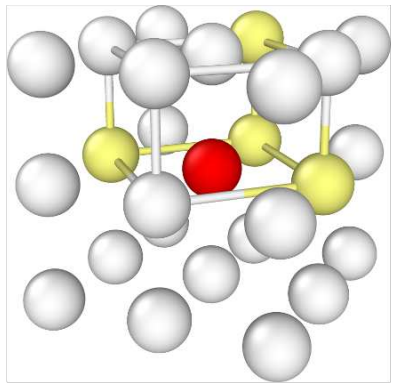

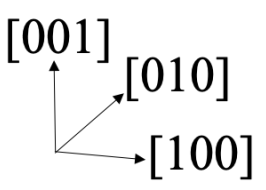

(d)

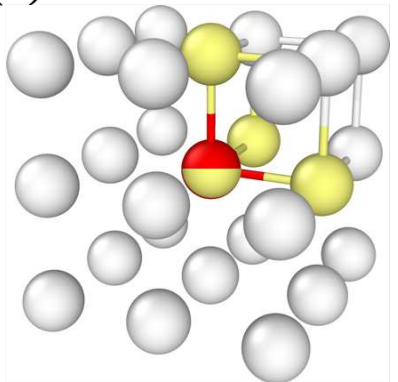

Figure 5. The three tetragonal unit cell in the (a) [100], (b) [010], and (c) [001] directions respectively, and (d)The cubic unit cell. Where the target atoms are in red and the three position vectors are in yellow.

The crystal variant corresponding to the unit cell can be identified when the theoretical transformation matrix of that variant is with the highest similarity to one of the four transformation matrices of the unit cell. The similarity was defined by the 15 conditions adopted and revised from Yang and Tsou ${ }^{17}$ and Lu et al. ${ }^{18}$ as shown in Table 2. Where $\delta$ represents the tolerance to neglect thermal vibration of atoms in MD simulation. Here the value of 0.06 was used. Note that only the upper triangle of the matrices was considered since the matrices are positive-definite and symmetric. We applied the 15 conditions to the calculated transformation matrices, giving four binary conditional judgment vectors with size $1 \times 15$. The four vectors then examined by the theoretical characteristic table (Table 3) listing the matched conditions corresponding to each variant/phase considered in the current work. The level of the similarity of each unit cell can be determined by counting the number of the matched conditions. The transformation matrix of the unit cell with the highest similarity with the theoretical one of the crystal variant can be correlated and classified. Note that whenever more than one crystal variant correlated to the unit cell or the highest similarity of the unit cell was less than 9 out of 15 conditions, the unit cell was classified as Other.

\begin{tabular}{c|c|c|c|c}
\hline I $: U_{11}-1 \geq \delta$ & IV $: U_{11}-1<-\delta$ & VII $: U_{12} \geq \delta$ & $\mathrm{X}: U_{12} \leq-\delta$ & $\mathrm{XIII}:\left\|U_{12}|-| U_{13}\right\| \leq 2 \delta$ \\
II $: U_{22}-1 \geq \delta$ & $\mathrm{V}: U_{22}-1<-\delta$ & $\mathrm{VIII}: U_{13} \geq \delta$ & $\mathrm{XI}: U_{13} \leq-\delta$ & $\mathrm{XIV}:\left\|U_{12}|-| U_{23}\right\| \leq 2 \delta$ \\
III $: U_{33}-1 \geq \delta$ & $\mathrm{VI}: U_{33}-1<-\delta$ & $\mathrm{IX}: U_{23} \geq \delta$ & $\mathrm{XII}: U_{23} \leq-\delta$ & $\mathrm{XV}:\left\|U_{13}|-| U_{23}\right\| \leq 2 \delta$ \\
\hline
\end{tabular}

Table 2. The 15 conditions for elements in the transformation matrix that can distinguish all the variants. 


\begin{tabular}{|c|c|c|}
\hline \multicolumn{3}{|l|}{$\begin{array}{l}\text { Austenite }(A) \\
A=\text { XIII, XIV, XV }\end{array}$} \\
\hline \multicolumn{3}{|l|}{ Orthorhombic $(O)$} \\
\hline$O 1=$ II, III, IV, IX, XIII & $O 2=\mathrm{II}, \mathrm{III}, \mathrm{IV}, \mathrm{XII}, \mathrm{XIII}$ & $O 3=\mathrm{I}, \mathrm{III}, \mathrm{V}, \mathrm{VIII}, \mathrm{XIV}$ \\
\hline$O 4=\mathrm{I}, \mathrm{III}, \mathrm{V}, \mathrm{XI}, \mathrm{XIV}$ & O5 = I, II, VI, VII, XV & $O 6=\mathrm{I}, \mathrm{II}, \mathrm{VI}, \mathrm{X}, \mathrm{XV}$ \\
\hline \multicolumn{3}{|l|}{$\operatorname{Monoclinic}(M)$} \\
\hline$M 1=\mathrm{II}, \mathrm{III}, \mathrm{IV}, \mathrm{IX}, \mathrm{X}, \mathrm{XI}, \mathrm{XIII}$ & $M 2=$ II, III, IV, VII, VIII, IX, XIII & M3 = II, III, IV, VII, XI, XII, XIII \\
\hline M4 = II, III, IV, VIII, X, XII, XIII & M5 = I, III, V, VIII, X, XII, XIV & $M 6=\mathrm{I}, \mathrm{III}, \mathrm{V}, \mathrm{VII}, \mathrm{VIII}, \mathrm{IX}, \mathrm{XIV}$ \\
\hline$M 7$ = I, III, V, VII, XI, XII, XIV & $M 8=\mathrm{I}, \mathrm{III}, \mathrm{V}, \mathrm{IX}, \mathrm{X}, \mathrm{XI}, \mathrm{XIV}$ & $M 9$ = I, III, VI, VII, XI, XII, XV \\
\hline$M 10=\mathrm{I}, \mathrm{III}, \mathrm{VI}, \mathrm{VII}, \mathrm{VIII}, \mathrm{IX}, \mathrm{XV}$ & $M 11=\mathrm{I}, \mathrm{II}, \mathrm{VI}, \mathrm{IX}, \mathrm{X}, \mathrm{XI}, \mathrm{XV}$ & $M 12$ = I, II, VI, VIII, X, XII, XV \\
\hline $\begin{array}{l}\text { Rhombohedral }(R) \\
R 1=\text { IV, V, VI, X, XI, XII, XIII, XIV, XV }\end{array}$ & $R 2=\mathrm{IV}, \mathrm{V}, \mathrm{VI}, \mathrm{VII}, \mathrm{IX}, \mathrm{XI}, \mathrm{XIII}, \mathrm{XIV}, \mathrm{XV}$ & $R 3=\mathrm{IV}, \mathrm{V}, \mathrm{VI}, \mathrm{VIII}, \mathrm{IX}, \mathrm{X}, \mathrm{XIII}, \mathrm{XIV}, \mathrm{XV}$ \\
\hline$R 4=\mathrm{IV}, \mathrm{V}, \mathrm{VI}, \mathrm{VII}, \mathrm{VIII}, \mathrm{XII}, \mathrm{XIII}, \mathrm{XIV}, \mathrm{XV}$ & & \\
\hline
\end{tabular}

Table 3. The required conditions listed in Table 2 for each crystal variant in the austenite and martensite phases.

For example, a given transformation matrix $\mathbf{U}$ corresponding to one of the four unit cells obtained from a typical SMA MD calculation and the arrangement of theoretical transformation matrix $\mathbf{U}_{O 6}$ are shown as follows

$$
\mathbf{U}=\left[\begin{array}{ccc}
1.04 & -0.055 & 0.0033 \\
-0.055 & 1.038 & -0.0011 \\
0.0033 & -0.0011 & 0.944
\end{array}\right], \mathbf{U}_{O 6}=\left[\begin{array}{ccc}
\alpha & -\beta & 0 \\
-\beta & \alpha & 0 \\
0 & 0 & \gamma
\end{array}\right]
$$

where $\alpha, \beta, \gamma$ are material constants. We examined the components of $\mathbf{U}$ by Table 2 and 3 . It can be observed that $\mathbf{U}$ and $\mathbf{U}_{O 6}$ are with the highest level of similarity, as all of the 15 conditions are matched, while other transformation matrices of the remaining three unit cells had lower levels of similarity. Thus, we classified the unit cell with the transformation matrix $\mathbf{U}$ as crystal variant $O_{6}$. This procedure looped throughout all the unit cells in the model, giving a rough classification of crystal variant. However, in a uniform domain of a given variant, it may occur that a single unit cell was classified as Other, due to the nature of thermal and dynamic fluctuation in MD calculations. This phenomenon is, in fact, not energetically favorable in the reality.

Thus, in the current work, a regional voting strategy was introduced to classify these singular unit cells. All the unit cell which were classified as Other and with similarity higher than $9 / 15$ corresponding to more than one crystal variant, were re-classified as the crystal variant of the majority of the surrounding unit cells. Where the surrounding unit cells are defined as the nearest 12 unit cells for the target singular unit cell, since the coordination number of HCP structure of orthorhombic and monoclinic phases is also 12. The same number is used in $\mathrm{CNA}^{13}$. Note that this procedure should repeat several times to minimize the number of Other in the model. In this way, the detail of the switching between the crystal variants and the corresponding microstructural evolution generated by molecular dynamics simulation can be revealed.

\subsection{Graph Neural Network and Model setting}

In the current work, GraphSAGE model ${ }^{24}$ is used. Similar with CNN, high efficiency and accuracy of prediction can be achieved as the model enables the local connection between nodes specified by 
the adjacency matrix and weight sharing in the same layer. The range of the local connection can be specified by the depth of the layers. Moreover, GraphSAGE can process both euclidean and non-euclidean structure of data with the help of topological graph format of data. Aggregators which aggregate the neighboring information to the target vertex, were adopted in the model enabling inductive learning, better generalization ability, and high efficiency for large-scale graph data.

The current MVIM-G model adopted two layers of GraphSAGE with the dropout technique to aggregate the information of the second neighboring atoms. The learning rate is set as 0.006. The loss function used here is Cross-Entropy Loss. Adam ${ }^{34}$ optimizer with weight decay mechanisms was adopted in the model. Here three models with the same setting were trained individually and their predictions then ensembled to obtain the final decision of the classification of the variants.

\subsubsection{Data Preparation for model training}

The dataset preparation for the training of graph neural network can be divided into two parts. The first is the graph $G(V, E)$ that can express the connection relationship of all vertices in the space, i.e. the position of each atom. The second is the feature matrix that incorporates the features of each vertex, i.e. the distortion of the corresponding unit cell. As mentioned in section 4.1, the distortion of the target atom's four reference unit cells can be described by the relative displacement of the seven adjacent atoms (i.e. the yellow atoms shown in Fig. 5) before and after the phase transformation. Thus, the graph structure data $G(V, E)$ used in the current study is in the size of $(N, 7)$, where $N$ is the number of the atoms in the considered space. Then, the feature vectors of the seven neighboring atoms for $N$ target atoms concatenate into a feature matrix with size of $(N, 21)$.

To enable a good applicability of the model for the classification of different crystal variants/phases, the training data set needs to cover a variety of cases and the number of distinct variants is required to be balanced. Thus, the cases of MD simulation were tailored to cover various boundary conditions including different force fields, temperature- and stress-induced martensitic transformation, as shown in Table 4. In Case 1 to Case 5, all simulated ensemble settings are NPT. Case 1 and Case 2 adopted different force fields and temperatures. The crystal was subjected to a ramped force from 1.013 to 30000 bars. Case 3 to 5 is a temperature-induced simulation with a two-axis coupling boundary settings, including xy, xz, yz axes. The data generated by Case 3 to 5 allows the current model learn the correspondence between the occurred variants and the orientation of the deformation.

Note that $\mathrm{R}$ phases are relatively rare in both the reality and molecular simulations, and thus, it is challenging to generate a sufficient amount of data for $\mathrm{R}$ phase by conventional atomic simulation. To balance the number of each category of phase/variant in the dataset, we generated 1 million artificial unit cell for each crystal variants in the $\mathrm{R}$ phase. Where $\pm 0.1 \%$ of lattice constant of random fluctuation was added to the generated unit cells, to imitating the thermal fluctuation of the lattice. Finally, we picked around 1 million data points for each crystal variant from the results of MD simulation as the training dataset. The entire training data set contains 113 independent MD snapshots and about 23 million atoms in total. 


\begin{tabular}{|l||c|c|c|c|}
\hline & Force field & Temperature $(\mathrm{K})$ & Force $($ bars $)$ & Coupled axes \\
\hline Case 1 & FS & 500 & $1.01325 \rightarrow 30000$ & NA \\
\hline Case 2 & MEAM & 325 & $1.01325 \rightarrow 30000$ & NA \\
\hline Case 3 & MEAM & $500 \rightarrow 100$ & 0 & {$[100]$ and [010] } \\
\hline Case 4 & MEAM & $500 \rightarrow 100$ & 0 & {$[100]$ and [001] } \\
\hline Case 5 & MEAM & $500 \rightarrow 100$ & 0 & {$[010]$ and [001] } \\
\hline
\end{tabular}

Table 4. The settings in the MD simulation for five cases of MD calculation to generate the training dataset.

\subsubsection{Data Preparation for model validation}

Two cases of temperature-induced and stress-induced martensitic transformation in SMAs that have not appeared in the training data were considered here, in order to demonstrate the versatility of the current MVIM-G model. 2NN-MEAM potential energy ${ }^{28}$ was adopted by the simulation for both cases. The temperature-induced model was controlled by the isothermal-isobaric canonical ensemble (NPT) with pressure of 1.013 bar. The periodic boundary condition (PBC) was applied in all directions. The model was firstly relaxed at $0 \mathrm{~K}$ by the conjugate gradient method to achieve energy minimization and dynamic equilibrium. Then the initial velocity of the atoms at $500 \mathrm{~K}$ was assigned by Gaussian distribution for thermal equilibrium. This is because $500 \mathrm{~K}$ is well above the austenite finish temperature for NiTi SMAs, enabling a stable initial structure of austenite phase. After thermal equilibrium, the model was cooled from $500 \mathrm{~K}$ to $100 \mathrm{~K}$, and then gradually heated from $100 \mathrm{~K}$ to $325 \mathrm{~K}$, resulting in a stable microstructure with martensite phases.

For the stress-induced case, a nanoindentation process which resulted in an unrecoverable plastic deformation at the surface of a NiTi crystal was simulated. Here NPT ensemble was used, and the energy minimization process was the same as it in the temperature-induced case. The periodic boundary condition was applied in [100] and [010] directions only, and the free surface was applied in the positive [001] direction. The temperature $325 \mathrm{~K}$ of thermal equilibrium is consistent with the temperature selected in the literature ${ }^{15}$, which could obtain a stable martensitic transformation between austenite and martensite phases. The indenter used here was with radius of $30 \AA$ located $3 \AA$ above the (001) plane to avoid the overlap of the indenter and the crystal. Next, the indenter moved downward with a constant rate (1.5x $10^{-4} \AA$ / femtosecond) until the indentation depth reaches $30 \AA$. Finally, the indenter was unloaded with the same rate. The results generated by the both cases were then post-processed by the CNA, MVIM, and the proposed MVIM-G model.

\section{References}

1. Hartl, D. J. \& Lagoudas, D. C. Aerospace applications of shape memory alloys. Proc. Inst. Mech. Eng. Part G: J. Aerosp. Eng. DOI: 10.1243/09544100JAERO211 (2007).

2. Concilio, A. \& Lecce, L. Shape Memory Alloy Engineering: For Aerospace, Structural and Biomedical Applications (2014).

3. Khoo, Z. X. et al. A review of selective laser melted NiTi shape memory alloy, DOI: 10.3390/ ma11040519 (2018).

4. Manfredi, L., Huan, Y. \& Cuschieri, A. Low power consumption mini rotary actuator with SMA wires. Smart Mater. Struct. DOI: 10.1088/1361-665X/aa8aa4 (2017). 
5. Ölander, A. An electrochemical investigation of solid cadmium-gold alloys. J. Am. Chem. Soc. DOI: 10.1021/ja01349a004 (1932).

6. Wayman, C. \& Duerig, T. An Introduction to Martensite and Shape Memory. Eng. Aspects Shape Mem. Alloy. DOI: 10.1016/b978-0-7506-1009-4.50005-6 (1990).

7. Chang, L. C. \& Read, T. A. Plastic Deformation and Diffusionless Phase Changes in Metals - the Gold-Cadmium Beta Phase. JOM DOI: 10.1007/bf03398954 (1951).

8. Perkins, J. Residual stresses and the origin of reversible (two-way) shape memory effects. Scripta Metall. DOI: 10.1016/0036-9748(74)90191-4 (1974).

9. Stoiber, J., Van Humbeeck, J. \& Gotthardt, R. Hysteresis Effects During Martensitic Transformation in $\mathrm{Cu}-\mathrm{Zn}-\mathrm{Al}$ Studied by Internal Friction Measurements. Mater. Sci. Forum DOI: 10.4028/www. scientific.net/msf.56-58.505 (1991).

10. Patoor, E., Lagoudas, D. C., Entchev, P. B., Brinson, L. C. \& Gao, X. Shape memory alloys, Part I: General properties and modeling of single crystals. Mech. Mater. 38, 391-429, DOI: 10.1016/j. mechmat.2005.05.027 (2006).

11. Honeycutt, J. D. \& Andersen, H. C. Molecular dynamics study of melting and freezing of small Lennard-Jones clusters. J. Phys. Chem. DOI: 10.1021/j100303a014 (1987).

12. Faken, D. \& Jónsson, H. Systematic analysis of local atomic structure combined with $3 \mathrm{D}$ computer graphics. Comput. Mater. Sci. DOI: 10.1016/0927-0256(94)90109-0 (1994).

13. Stukowski, A. Structure identification methods for atomistic simulations of crystalline materials. Model. Simul. Mater. Sci. Eng. DOI: 10.1088/0965-0393/20/4/045021 (2012). 1202.5005.

14. Larsen, P. M., Schmidt, S. \& SchiØtz, J. Robust structural identification via polyhedral template matching. Model. Simul. Mater. Sci. Eng. DOI: 10.1088/0965-0393/24/5/055007 (2016). 1603.05143.

15. Srinivasan, P., Nicola, L. \& Simone, A. Modeling pseudo-elasticity in NiTi: Why the MEAM potential outperforms the EAM-FS potential. Comput. Mater. Sci. DOI: 10.1016/j.commatsci.2017.03.026 (2017).

16. Otsuka, K. \& Kakeshita, T. Science and Technology of Shape Memory Alloys : New Developments. Mater. Res. Soc. Bull. DOI: 10.1557/mrs2002.43 (2002).

17. Yang, C. W. \& Tsou, N. T. Microstructural analysis and molecular dynamics modeling of shape memory alloys. Comput. Mater. Sci. DOI: 10.1016/j.commatsci.2017.02.011 (2017).

18. Lu, H. Y., Chen, C. H. \& Tsou, N. T. The analysis of superelasticity and microstructural evolution in NiTi single crystals by molecular dynamics. Materials DOI: 10.3390/ma12010057 (2018).

19. Chen, H. Y. \& Tsou, N. T. The analysis of thermal-induced phase transformation and microstructural evolution in Ni-Ti based shape memory alloys by molecular dynamics. CMES - Comput. Model. Eng. Sci. 120, 319-332, DOI: 10.32604/cmes.2019.06447 (2019).

20. $\mathrm{Xu}, \mathrm{K}$., Jegelka, S., Hu, W. \& Leskovec, J. How powerful are graph neural networks? In 7th International Conference on Learning Representations, ICLR 2019 (2019).

21. Zhou, Z. Graph Convolutional Networks for Molecules. In arXiv (2017). arXiv:1706.09916v1.

22. Liu, K. et al. Chemi-net: A molecular graph convolutional network for accurate drug property prediction. Int. J. Mol. Sci. DOI: 10.3390/ijms20143389 (2019). 
23. Coley, C. W. et al. A graph-convolutional neural network model for the prediction of chemical reactivity. Chem. Sci. DOI: 10.1039/c8sc04228d (2019).

24. Hamilton, W. L., Ying, R. \& Leskovec, J. Inductive representation learning on large graphs. In Advances in Neural Information Processing Systems (2017). 1706.02216.

25. Stukowski, A. Visualization and analysis of atomistic simulation data with OVITO-the Open Visualization Tool. Model. Simul. Mater. Sci. Eng. DOI: 10.1088/0965-0393/18/1/015012 (2010).

26. Waitz, T., Pranger, W., Antretter, T., Fischer, F. D. \& Karnthaler, H. P. Competing accommodation mechanisms of the martensite in nanocrystalline NiTi shape memory alloys. Mater. Sci. Eng. A DOI: 10.1016/j.msea.2007.03.122 (2008).

27. Otsuka, K. \& Ren, X. Physical metallurgy of Ti-Ni-based shape memory alloys, DOI: 10.1016/j. pmatsci.2004.10.001 (2005).

28. Lee, B. J. \& Baskes, M. I. Second nearest-neighbor modified embedded-atom-method potential. Phys. Rev. B - Condens. Matter Mater. Phys. DOI: 10.1103/PhysRevB.62.8564 (2000).

29. Buehler, W. J., Gilfrich, J. V. \& Wiley, R. C. Effect of Low-Temperature Phase Changes on the Mechanical Properties of Alloys near Composition TiNi. J. Appl. Phys. 34, 1475-1477, DOI: 10.1063/1.1729603 (1963).

30. Gao, Y. et al. An origin of functional fatigue of shape memory alloys. Acta Materialia 126, 389-400, DOI: 10.1016/j.actamat.2017.01.001 (2017).

31. Ericksen, J. L. On the Cauchy-Born rule. Math. Mech. Solids DOI: $10.1177 / 1081286507086898$ (2008).

32. Bhattacharya, K. Microstructure of martensite: why it forms and how it gives rise to the shape-memory effect. Choice Rev. Online DOI: 10.5860/choice.41-6554 (2004).

33. Chadwick, P. Continuum Mechanics: Concise Theory and Problems. Continuum Mech. Concise Theory Probl. 130, 20 (1998).

34. Kingma, D. P. \& Ba, J. L. Adam: A method for stochastic optimization. In 3rd International Conference on Learning Representations, ICLR 2015 - Conference Track Proceedings (2015). 1412. 6980.

\section{Acknowledgements}

The authors acknowledge the financial support of Ministry of Science and Technology Taiwan, Grant No MOST 107-2221-E-009-013-MY2 and MOST 109-2224-E-080-001. We thank National Center for High-Performance Computing of Taiwan for providing computational and storage resources.

\section{Author contributions statement}

Conceptualization, methodology, and writing, Y.-M.T. and N.-T.T.; data generation, Y.-M.T. and P.-T.W.; results analysis, N.-Y.C. and A.-C.Y.; supervision and project administration, N.-T.T. All authors reviewed the manuscript. 
Figures

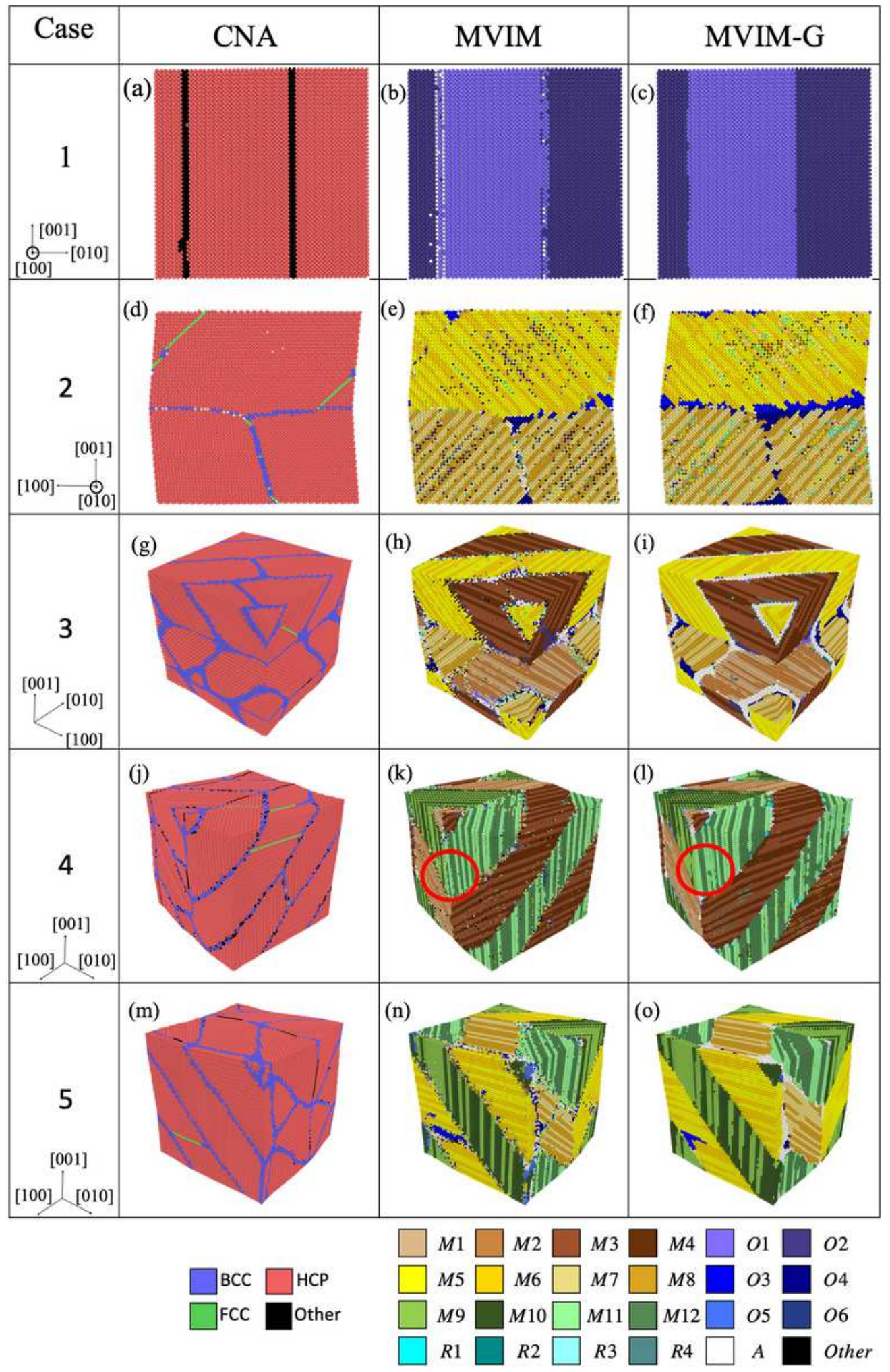

Figure 1

Selected snapshots for cases $1-5$ post-processed by CNA, MVIM and MVIM-G. 


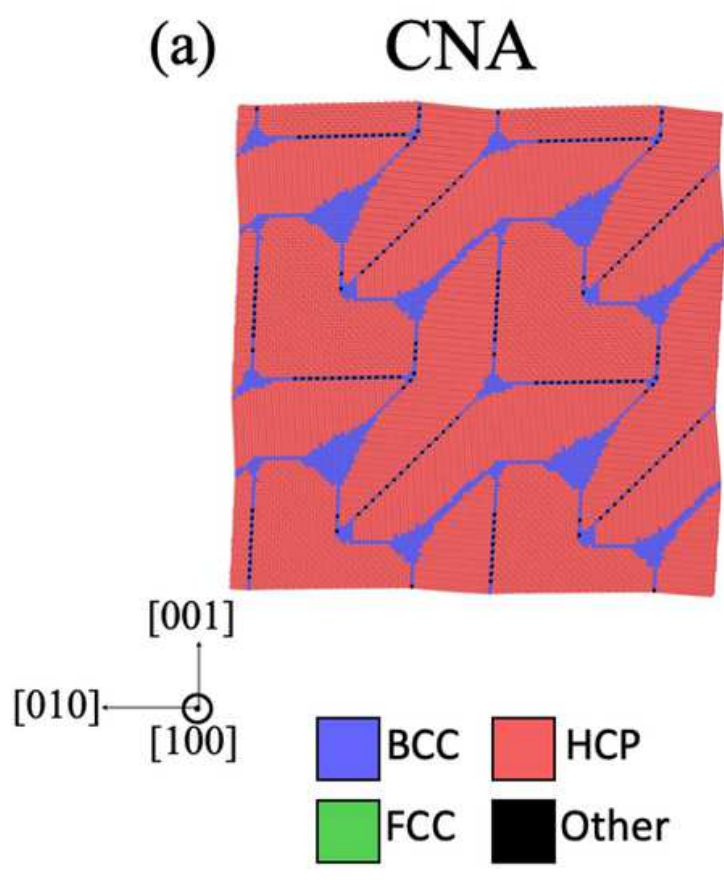

(b) MVIM
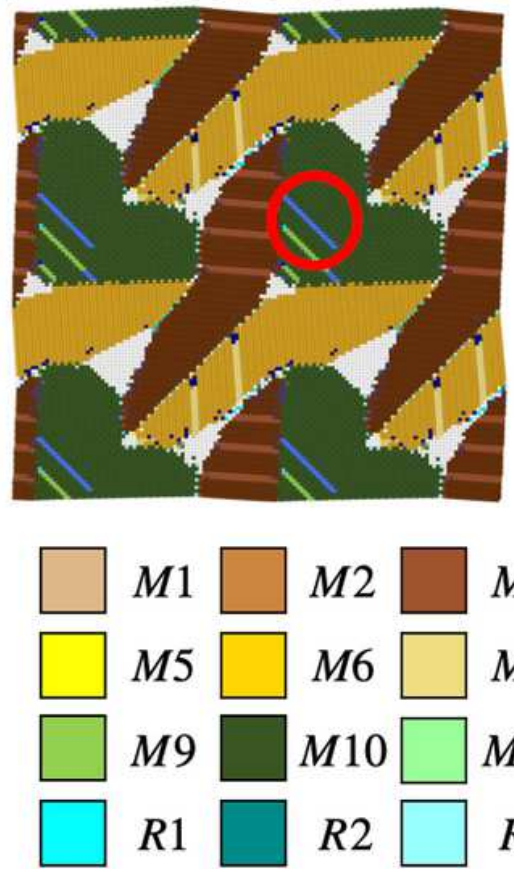

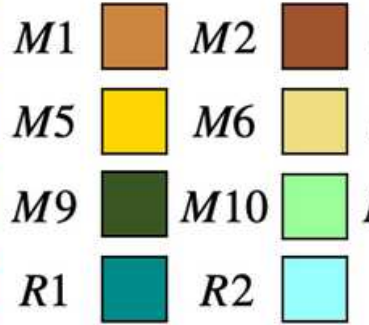

(c) MVIM-G

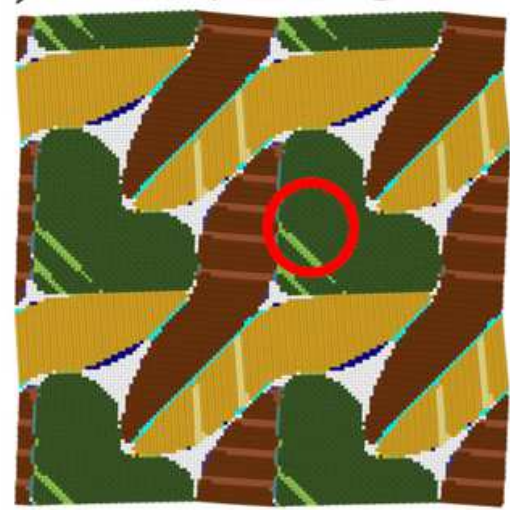

M3

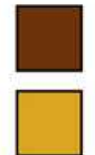

M4

$\square 01$

$\square 02$ $\begin{array}{lll}M 8 & & 03 \\ & & \end{array}$ $M 12 \square 05 \square 06$ $R 4 \square A$

Figure 2

Selected snapshots for the case of isobaric temperature-induced martensitic transformation at $325 \mathrm{~K}$ post-processed by CNA, MVIM and MVIM-G. 


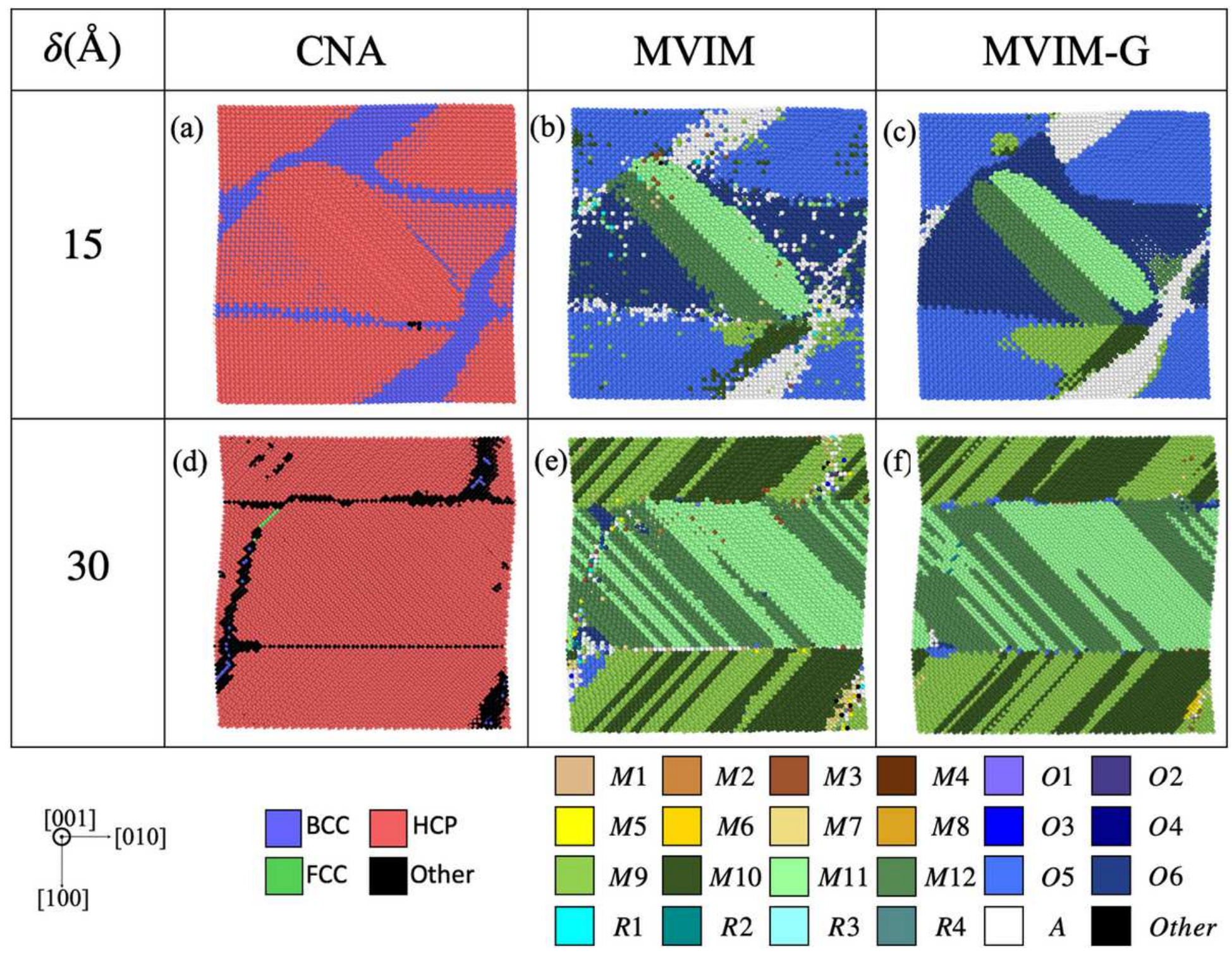

Figure 3

Selected snapshots at the cross-section plane of (001) for the case of nanoindentation when the indent depth $\delta=15 ; 30 \AA$ A post-processed by CNA, MVIM and MVIM-G. 


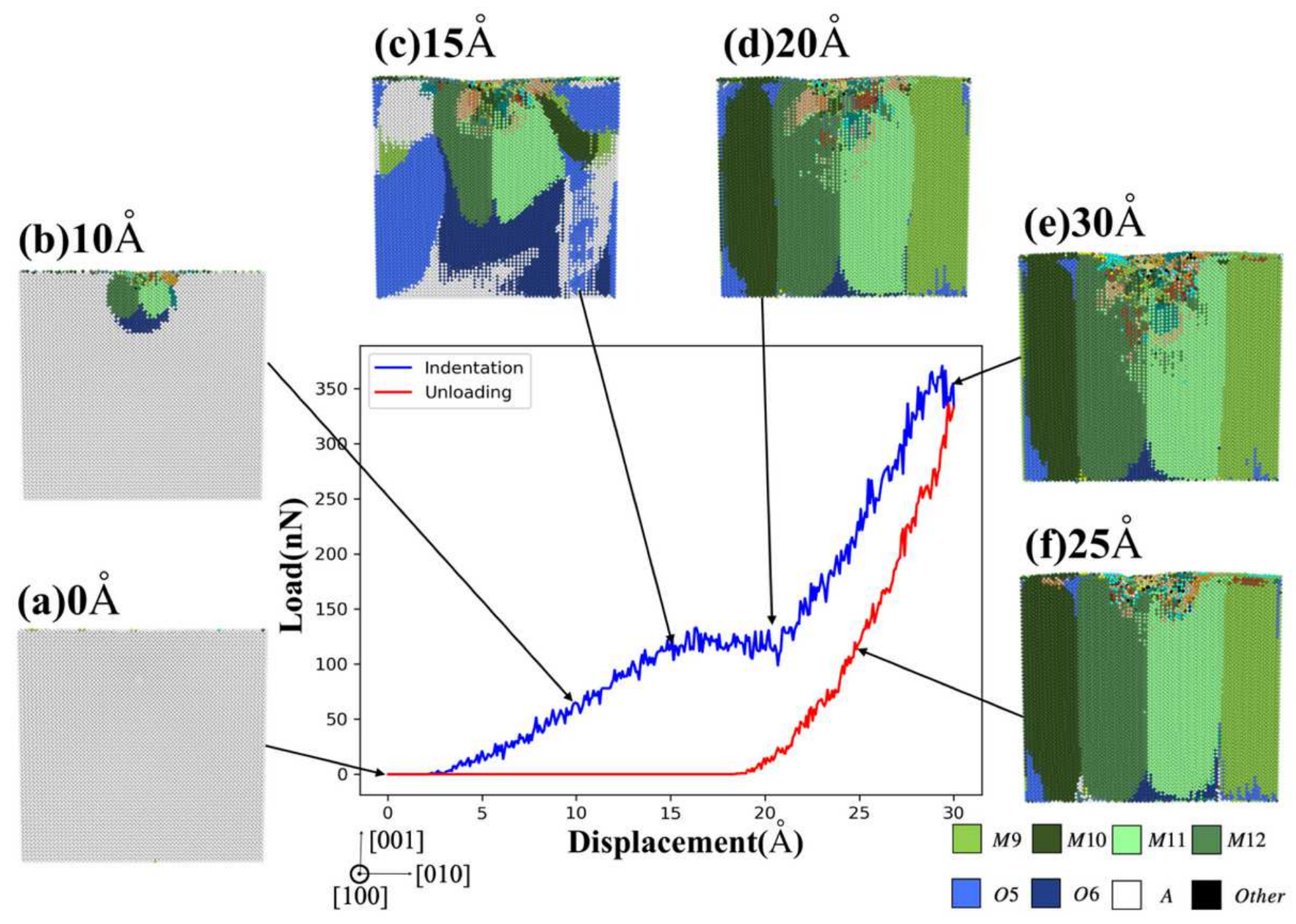

Figure 4

The P-L curve of NiTi SMAs during the nanoindentation process. The corresponding microstructures on the cross-section plane of (100) are post-processed by the MVIM-G.

(a)

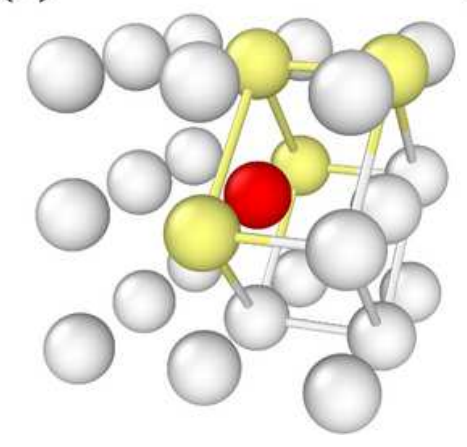

(b)

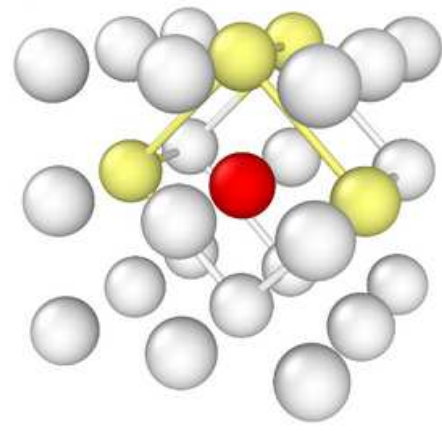

(c)

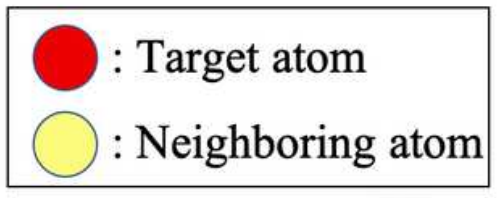

(d)

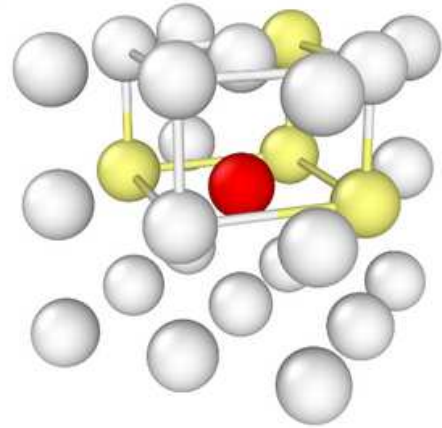

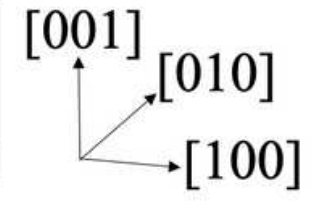

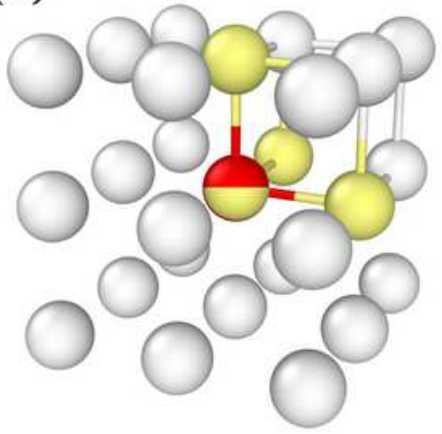


The three tetragonal unit cell in the (a) [100], (b) [010], and (c) [001] directions respectively, and (d)The cubic unit cell. Where the target atoms are in red and the three position vectors are in yellow. 